\title{
Threshold dynamics of an SEAIR epidemic model with ap- plication to COVID-19
}

\author{
Zijun Zheng, Youping Yang* \\ School of Mathematics and Statistics, Shandong Normal University, Jinan, 250014, P. R. China.
}

\begin{abstract}
In this paper, a Susceptible-Exposed-Asymptomatic-Infectious-Recovered (SEAIR) epidemic model with application to COVID-19 is established by capturing the key features of the disease. The global dynamics of the model is analyzed by constructing appropriate Lyapunov functions utilizing the basic reproduction number $R_{0}$ as an index. We obtain that when $R_{0}<1$, the disease-free equilibrium is globally asymptotically stable. While for $R_{0}>1$, the endemic equilibrium is globally asymptotically stable. Furthermore, we consider the pulse vaccination for the disease and give an impulsive differential equations model. The definition of the basic reproduction number $R_{0}$ of this system is given by utilizing the next generation operator. By the comparison theorem and persistent theory, we obtain that when $\mathrm{R}_{0}<1$, the disease-free periodic solution is globally asymptotically stable. Otherwise, the disease will persist and there will be at least one nontrivial periodic solution. Numerical simulations to verify our conclusions are given at the end of each of these theorems.
\end{abstract}

Keywords: COVID-19, SEAIR, Lyapunov function, global stability, pulse vaccination, persistent theory.

2020 MSC: 34C60, 37C75, 92B05.

(C)2022 All rights reserved.

\section{Introduction}

The ongoing COVID-19 is the largest pandemic worldwide nowadays. Researches about the virus and how the virus influences the infected persons are still in progress [5-9, 14, 23, 26]. However, an important observation is that the incubation period of the virus ranges from 1 to 14 days with an approximate median 5 days [19], and individuals in incubation period can also carry the virus and infect others. Another key feature is that an infected person can spread the disease before showing any symptoms [13]. Therefore, individuals in the incubation period and those infected but asymptomatic are hidden virus carriers and disease spreaders, whose presence is a great threat and challenge for disease control and healthcare capacity buildup or the relaxation of lockdown measures [10].

A large number of mathematical models have so far been publicly released to study the transmission and control of the disease, which are built according to capturing the key features of COVID-19 [10, 12, 29, 30] to name a few. The incubation stage is addressed by considering an exposed compartment $E[29,30]$, however, all these models assume E carries the pathogen but cannot infect others. While the address of

\footnotetext{
*Corresponding author

Email address: yyang@sdnu.edu.cn (Youping Yang)
}

doi: $10.22436 /$ jnsa.015.02.05

Received: 2021-09-26 Revised: 2021-10-17 Accepted: 2021-11-19 
absence or presence of symptoms varies. In study [29], the infected individuals are accounted in separate classes $A$ and I with detected asymptomatically or symptomatically. In the analysis performed in [11], the authors considered three different severity levels of infected, including prodromic, asymptomatic and symptomatic phases. Then what we intend to do in this paper is to establish a SEAIR epidemic model with considering the infectiveness of the exposed class and the infected with or without symptoms based on the two main features of the disease.

In epidemiological models global stability is more appreciated in policy making. The experienced and successful method in establishing global stability is to construct appropriate Lyapunov function [16, 17, 32] and apply LaSalle's invariance principle [18]. There definitely exists a Lyapunov function if the equilibrium is globally stable $[15,21]$. However, how to choose the exact expression of the Lyapunov function, and further how to illustrate that the derivative of the Lyapunov function along the solutions of the model is negative definite or semidefinite remain challenged. In this paper, we apply an algebraic approach [22] to determine the coefficients of the combinations $u-u^{*}-u^{*} \ln \frac{\mathfrak{u}}{\mathfrak{u}^{*}}$, and further to express the derivative of the Lyapunov function as sums of the form $m_{k}\left(n_{k}-x_{k, 1}-x_{k, 2}-\cdots-x_{k, n_{k}}\right)$, where $m_{k} \geqslant 0, x_{k, i}, i=1,2, \ldots, n_{k}$, satisfies $\prod_{i=1}^{n_{k}} x_{k, i}=1$. Then by the property that the arithmetic mean is greater than or equal to the geometric mean, we can obtain that the derivative of the Lyapunov function along the solutions of the model is negative.

Immunization is the most economical and effective measure that benefits the entire human race from a global perspective and the history of human development. Continuous vaccination [27] requires that at least $95 \%$ of children should be immunized shortly after birth. In fact, it is difficult and expensive to implement vaccination for such a wide population coverage. Therefore, pulse vaccination to vaccinate a proportion of the susceptible at some intervals is more advantageous. The COVID-19 vaccine currently being developed and put into use is a preventive one. According to the key features of the disease, we establish a dynamic model with pulse vaccination [27, 28]. By using persistent theory [33, 36] and the comparison theorem of impulse differential equations $[33,36]$, we analyze the persistence of the disease by defining the basic reproduction number as the next infection operator.

The rest of this paper is organized as follows. In Section 2, an SEAIR epidemic model with application to COVID-19 is formulated. In Sections 3, the global asymptotic stability of the disease-free and the endemic equilibria is derived. In Sections 4, we consider the model of COVID-19 with pulse vaccination and analyze its persistence. We conclude the work in Section 5.

\section{Model formulation}

The populations are stratified as susceptible $(S(t))$, exposed $(E(t))$, infectious but asymptomatic $(A(t))$, infectious with symptoms $(I(t))$ and recovered $(R(t))$, based on the two key features of COVID-19. Then one has the following SEAIR compartmental model:

$$
\begin{aligned}
S^{\prime} & =\wedge-\beta S(I+\theta A+\nu E)-\mu S, \\
E^{\prime} & =\beta S(I+\theta A+\nu E)-(\delta+\mu) E, \\
A^{\prime} & =(1-p) \delta E-\left(\gamma_{1}+\alpha+\mu\right) A, \\
I^{\prime} & =p \delta E+\alpha A-\left(\gamma_{2}+d+\mu\right) I,
\end{aligned}
$$

and $R^{\prime}=\gamma_{1} A+\gamma_{2} I-\mu R$. The variable $R$ can be decoupled from the first four equations, hence we omit it in the following analysis. ' is the derivative with respect to time $t, \Lambda$ is the (constant) recruitment rate, $\beta$ is the transmission coefficient, $\theta$ and $v$ are the transmission coefficient regulators of asymptomatic $A$ and exposed $E$, respectively, $\mu$ is the natural death rate, while $d$ is the disease-induced death rate, $\alpha$ is the rate from asymptomatic to symptomatic infected persons, $\delta$ is the rate of exposed individuals to the infected compartment, $p$ is the probability of having symptoms among infected individuals, and $\gamma_{1}$ and $\gamma_{2}$ are the recovery rates of asymptomatic and symptomatic infected persons, respectively. 
Summing equations in (2.1) yields

$$
(S+E+A+I)^{\prime}=\Lambda-\mu(S+E+A+I)-\gamma_{1} A-\gamma_{2} I-d I \leqslant \Lambda-\mu(S+E+A+I),
$$

hence, the solutions of system (2.1) are uniformly and ultimately bounded, i.e., $S(t)+E(t)+A(t)+I(t) \leqslant$ $\frac{\Lambda}{\mu}$. Therefore, in the following, the dynamics of system (2.1) will be analyzed in a bounded feasible region

$$
\Gamma=\left\{X=(S, E, A, I) \in R_{+}^{4}: S(t)+E(t)+A(t)+I(t) \leqslant \frac{\Lambda}{\mu}\right\} .
$$

The basic reproduction number $R_{0}$ of system (2.1) can be obtained by applying the next generation matrix method [31], which is

$$
\begin{aligned}
R_{0} & =\rho\left(\left(\begin{array}{ccc}
v \beta S_{0} & \theta \beta S_{0} & \beta S_{0} \\
0 & 0 & 0 \\
0 & 0 & 0
\end{array}\right)\left(\begin{array}{ccc}
\delta+\mu & 0 & 0 \\
-(1-p) \delta & \gamma_{1}+\alpha+\mu & 0 \\
-p \delta & -\alpha & \gamma_{2}+d+\mu
\end{array}\right)^{-1}\right) \\
& =S_{0}\left[\frac{v \beta}{\delta+\mu}+\frac{(1-p) \delta \theta \beta}{\left(\gamma_{1}+\alpha+\mu\right)(\delta+\mu)}+\frac{p \delta \beta}{\left(\gamma_{2}+d+\mu\right)(\delta+\mu)}+\frac{\alpha(1-p) \delta \beta}{\left(\gamma_{1}+\alpha+\mu\right)\left(\gamma_{2}+d+\mu\right)(\delta+\mu)}\right],
\end{aligned}
$$

where $\rho$ is the spectral radius of a matrix and $S_{0}=\frac{\Lambda}{\mu}$.

Theorem 2.1. If the basic reproduction number $\mathrm{R}_{0}<1$, system (2.1) only has a disease-free equilibrium $\mathrm{E}_{0}=$ $\left(\frac{\Lambda}{\mu}, 0,0,0\right)$; while if $\mathrm{R}_{0}>1$, system (2.1) admits a disease-free equilibrium $\mathrm{E}_{0}$ and a unique endemic equilibrium $\mathrm{E}_{1}=\left(\mathrm{S}^{*}, \mathrm{E}^{*}, \mathrm{~A}^{*}, \mathrm{I}^{*}\right)$ given by

$$
S^{*}=\frac{\Lambda}{\mu R_{0}}, \quad E^{*}=\frac{\mu\left(R_{0}-1\right)}{(\delta+\mu) R_{0}}, \quad A^{*}=\frac{\mu \delta\left(R_{0}-1\right)(1-p)}{\left(\gamma_{1}+\alpha+\mu\right)(\delta+\mu) R_{0}}, \quad I^{*}=\frac{\mu \delta\left(R_{0}-1\right)\left[\left(\gamma_{1}+\alpha+\mu\right) p+\alpha(1-p)\right]}{\left(\gamma_{1}+\alpha+\mu\right)\left(\gamma_{2}+d+\mu\right)(\delta+\mu) R_{0}} .
$$

Proof. It can be easily seen that system (2.1) always has a disease-free equilibrium $E_{0}=\left(\frac{\Lambda}{\mu}, 0,0,0\right)$. Set the right-hand side of system (2.1) to be zero. Then, by the third and forth equations of system (2.1), one can obtain

$$
E=\frac{\gamma_{1}+\alpha+\mu}{(1-p) \delta} A, \quad I=\left(\frac{p}{1-p} \frac{\gamma_{1}+\alpha+\mu}{\gamma_{2}+\alpha+\mu}+\frac{\alpha}{\gamma_{2}+\alpha+\mu}\right) A
$$

By the second equation of system (2.1), one can obtain

$$
\begin{aligned}
S=\frac{(\delta+\mu) E}{\beta(I+\theta A+v E)} & =\frac{(\delta+\mu)\left(\gamma_{1}+\alpha+\mu\right)}{(1-p) \delta \beta\left(\frac{p}{1-p} \frac{\gamma_{1}+\alpha+\mu}{\gamma_{2}+\alpha+\mu}+\frac{\alpha}{\gamma_{2}+\alpha+\mu}+\theta+\frac{v\left(\gamma_{1}+\alpha+\mu\right)}{(1-p) \delta}\right)} \\
& =\frac{(\delta+\mu)\left(\gamma_{1}+\alpha+\mu\right)}{\frac{\delta \beta p\left(\gamma_{1}+\alpha+\mu\right)}{\gamma_{2}+\alpha+\mu}+\frac{(1-p) \delta \beta \alpha}{\gamma_{2}+\alpha+\mu}+(1-p) \delta \beta \theta+\nu \beta\left(\gamma_{1}+\alpha+\mu\right)}=\frac{\Lambda}{\mu} \frac{1}{R_{0}} .
\end{aligned}
$$

On the other hand,

$$
I+\theta A+v E=\left(\frac{p\left(\gamma_{1}+\alpha+\mu\right)}{(1-p)\left(\gamma_{2}+\alpha+\mu\right)}+\frac{\alpha}{\gamma_{2}+\alpha+\mu}+\frac{v\left(\gamma_{1}+\alpha+\mu\right)}{(1-p) \delta}\right) A,
$$

by the first equation of system (2.1), there also holds

$$
I+\theta A+v E=\frac{\Lambda-\mu S}{\beta S}=\frac{\mu}{\beta}\left(R_{0}-1\right) .
$$

Hence, the expression for A can be obtained. According to the relationship between $E$, I with $A$, we can obtain the expression of the endemic equilibrium, which exists when the basic reproduction number is larger than 1 . This finishes the proof. 


\section{Threshold dynamics}

3.1. Global asymptotic stability of the disease-free equilibrium

Theorem 3.1. If the basic reproduction number $\mathrm{R}_{0}<1$, then the disease-free equilibrium $\mathrm{E}_{0}=\left(\frac{\Lambda}{\mu}, 0,0,0\right)$ is globally asymptotically stable.

Proof.

(i) Firstly, we show that $E_{0}$ is locally stable by linearizing system (2.1) at $E_{0}$. The Jacobian matrix of system (2.1) at $\mathrm{E}_{0}$ reads

$$
J\left(E_{0}\right)=\left(\begin{array}{cccc}
-\mu & -\beta \nu \frac{\Lambda}{\mu} & -\beta \theta \frac{\Lambda}{\mu} & -\beta \frac{\Lambda}{\mu} \\
0 & \beta v \frac{\Lambda}{\mu}-(\delta+\mu) & \beta \theta \frac{\Lambda}{\mu} & \beta \frac{\Lambda}{\mu} \\
0 & (1-p) \delta & -\left(\gamma_{1}+\alpha+\mu\right) & 0 \\
0 & p \delta & \alpha & -\left(\gamma_{2}+d+\mu\right)
\end{array}\right)
$$

Then the characteristic equation can be expressed as

$$
w_{0}(\lambda)=\left|\lambda H-J\left(E_{0}\right)\right|=(\lambda+\mu) f(\lambda)=0,
$$

where $H$ is a $4 \times 4$ identity matrix, and $f(\lambda)=\lambda^{3}+a_{1} \lambda^{2}+a_{2} \lambda+a_{3}$ with

$$
\begin{aligned}
& a_{1}=a+b+c \\
& a_{2}=a b+\left(b c-\beta p \delta \frac{\Lambda}{\mu}\right)+\left(a c-(1-p) \delta \beta \theta \frac{\Lambda}{\mu}\right), \\
& a_{3}=a b c-\alpha \beta(1-p) \delta \frac{\Lambda}{\mu}-\beta p \delta a \frac{\Lambda}{\mu}-(1-p) \delta \beta \theta b \frac{\Lambda}{\mu}
\end{aligned}
$$

and

$$
a=\left(\gamma_{1}+\alpha+\mu\right), b=\left(\gamma_{2}+d+\mu\right), c=-\left\{\beta v \frac{\Lambda}{\mu}-(\delta+\mu)\right\} .
$$

Since $R_{0}<1$, then $a, b$ and $c$ are all positive values. Obviously, the characteristic equation has a negative eigenvalue $-\mu$, then what we need to do is to explore the roots of $f(\lambda)=0$. It can be easily seen $a_{1}>0$. Also, $a_{2}>a b>0$ and $a_{3}>0$ hold since $R_{0}<1$. According to the Descartes' Sign Rules, $f(\lambda)=0$ has three negative real roots. What follows is all the eigenvalues of the characteristic equation (3.1) are negative. Hence, $E_{0}$ is locally stable.

(ii) Secondly, we illustrate that $E_{0}$ is globally attractive by constructing suitable Lyapunov function. Define a Lyapunov function

$$
V(t)=\left(S-S_{0} \ln S\right)+E+A+I .
$$

The derivative of $V(t)$ along solutions of system (2.1) is

$$
\begin{aligned}
\frac{d V}{d t}= & \left(1-\frac{S_{0}}{S}\right) S^{\prime}+E^{\prime}+A^{\prime}+I^{\prime} \\
= & \left(1-\frac{S_{0}}{S}\right)[\Lambda-\beta S(I+\theta A+v E)-\mu S]+\beta S(I+\theta A+\nu E)-(\delta+\mu) E+(1-p) \delta E \\
& -\left(\gamma_{1}+\mu\right) A+p \delta E+\alpha A-\left(\gamma_{2}+d+\mu\right) I \\
= & \left(1-\frac{S_{0}}{S}\right)(\Lambda-\mu S)-S_{0} \beta(I+\theta A+\nu E)-\mu E-\left(\gamma_{1}+\mu\right) A-\left(\gamma_{2}+d+\mu\right) I \\
= & \Lambda\left(1-\frac{S_{0}}{S}\right)+\mu S_{0}\left(1-\frac{S}{S_{0}}\right)-S_{0} \beta(I+\theta A+\nu E)-\mu E-\left(\gamma_{1}+\mu\right) A-\left(\gamma_{2}+d+\mu\right) I
\end{aligned}
$$




$$
=\Lambda\left(2-\frac{S_{0}}{S}-\frac{S}{S_{0}}\right)-S_{0} \beta(I+\theta A+\nu E)-\mu E-\left(\gamma_{1}+\mu\right) A-\left(\gamma_{2}+d+\mu\right) I \leqslant 0 .
$$

Obviously, the singleton $E_{0}$ is the largest invariant set in $\left\{x \in \Gamma \mid \frac{d V}{d t}=0\right\}$. The global attractivity of $E_{0}$ is then inducted by the LaSalle's invariance principle [18]. Local stability and global attractivity lead to the global asymptotic stability of $\mathrm{E}_{0}$.
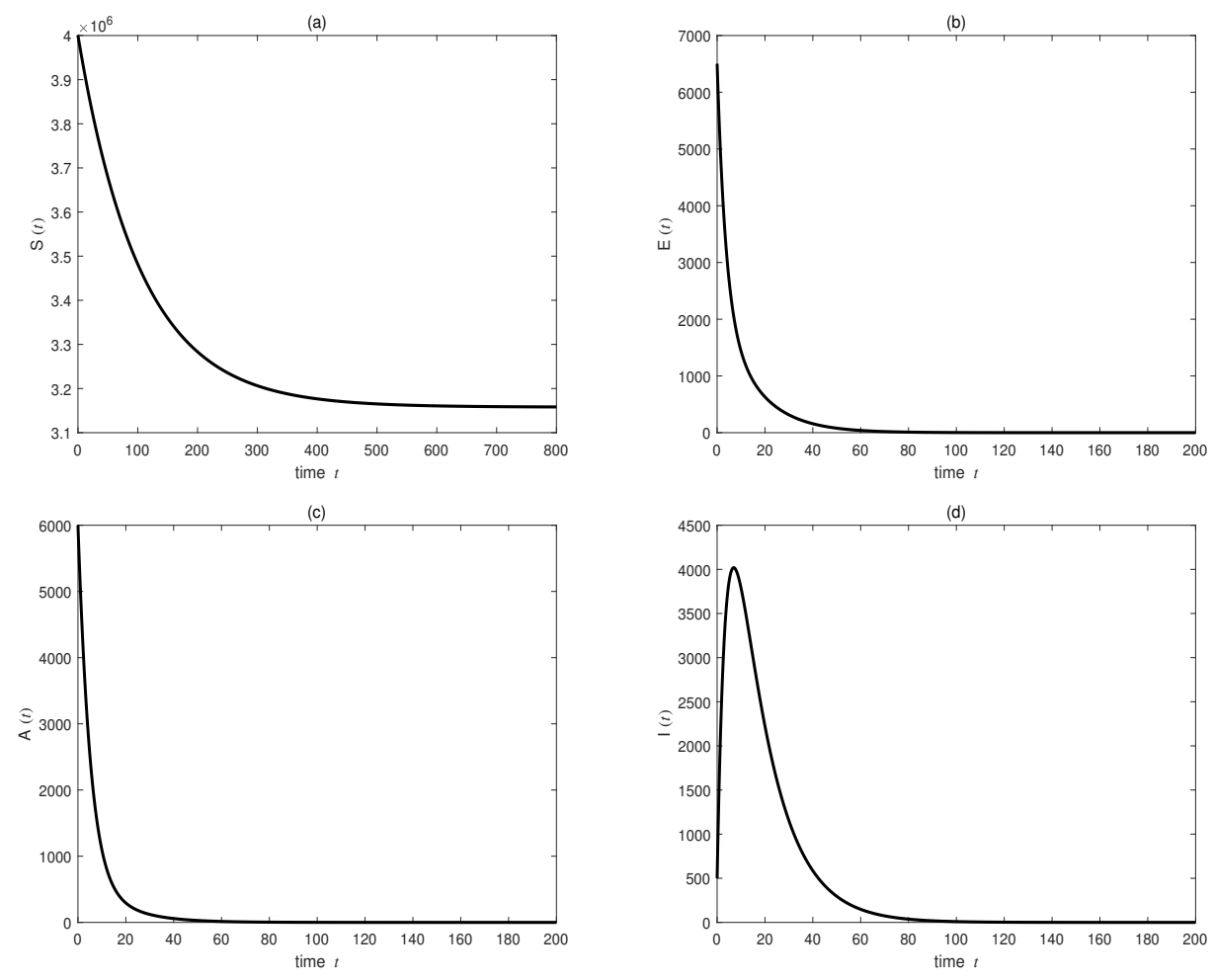

Figure 1: The disease-free equilibrium $\mathrm{E}_{0}$ is globally asymptotically stable when $\mathrm{R}_{0}=0.2210<1 . \Lambda=30000 ; \delta=0.2 ; \nu=0.032$; $\theta=0.045 ; \mu=0.0095 ; p=0.69 ; \gamma_{1}=0.1292 ; \alpha=0.1 ; \gamma_{2}=0.0978 ; d=0.008 ;$ and $\beta=0.00000001$. $S_{0}=3157894.73684$.

As is shown in Fig. 1, when the basic reproduction number $R_{0}<1$, the disease-free equilibrium $E_{0}$ is globally asymptotically stable.

\subsection{Global asymptotic stability of the endemic equilibrium}

Theorem 3.2. If $\mathrm{R}_{0}>1$, the endemic equilibrium $\mathrm{E}_{1}=\left(\mathrm{S}^{*}, \mathrm{E}^{*}, \mathrm{~A}^{*}, \mathrm{I}^{*}\right)$ is globally asymptotically stable.

Proof.

(i) Firstly, we show that $E_{1}$ is locally stable by linearizing system (2.1) at $E_{1}$. The Jacobian matrix of system (2.1) at $E_{1}$ reads

$$
J\left(E_{1}\right)=\left(\begin{array}{cccc}
-\mu R_{0} & -\beta v \frac{\Lambda}{\mu R_{0}} & -\beta \theta \frac{\Lambda}{\mu R_{0}} & -\beta \frac{\Lambda}{\mu R_{0}} \\
\mu\left(R_{0}-1\right) & \beta v \frac{\Lambda}{\mu R_{0}}-(\delta+\mu) & \beta \theta \frac{\Lambda}{\mu R_{0}} & \beta \frac{\Lambda}{\mu R_{0}} \\
0 & (1-p) \delta & -\left(\gamma_{1}+\alpha+\mu\right) & 0 \\
0 & p \delta & \alpha & -\left(\gamma_{2}+d+\mu\right)
\end{array}\right)
$$

Then the characteristic equation can be expressed as

$$
w_{1}(\lambda)=\left|\lambda H-J\left(E_{1}\right)\right|=\lambda^{4}+b_{1} \lambda^{3}+b_{2} \lambda^{2}+b_{3} \lambda+b_{4},
$$


where

$$
\begin{aligned}
\mathrm{b}_{1}= & \mu \mathrm{R}_{0}+\mathrm{a}+\mathrm{b}+\mathrm{l}, \\
\mathrm{b}_{2}= & \mu \mathrm{R}_{0}(\mathrm{a}+\mathrm{b}+\mathrm{l})+(\mathrm{al}+\mathrm{ab}+\mathrm{bl})-\mathrm{p} \delta \beta \frac{\Lambda}{\mu \mathrm{R}_{0}}-(1-p) \theta \delta \beta \frac{\Lambda}{\mu \mathrm{R}_{0}}+\frac{\beta \Lambda v\left(\mathrm{R}_{0}-1\right)}{\mathrm{R}_{0}}, \\
\mathrm{~b}_{3}= & \mu \mathrm{R}_{0}\left[(\mathrm{al}+\mathrm{ab}+\mathrm{bl})-\mathrm{p} \delta \beta \frac{\Lambda}{\mu \mathrm{R}_{0}}-(1-p) \theta \delta \beta \frac{\Lambda}{\mu \mathrm{R}_{0}}\right]+\mathrm{abl}-\alpha(1-p) \delta \beta \frac{\Lambda}{\mu \mathrm{R}_{0}}-\mathrm{p} \delta \beta \frac{\Lambda}{\mu \mathrm{R}_{0}} \mathrm{a} \\
& -(1-p) \theta \delta \beta \frac{\Lambda}{\mu \mathrm{R}_{0}} \mathrm{~b}+\frac{\beta \Lambda\left(\mathrm{R}_{0}-1\right)}{\mathrm{R}_{0}}[(\mathrm{a}+\mathrm{b}) v+\mathrm{p} \delta+(1-p) \theta \delta], \\
\mathrm{b}_{4}= & \mu \mathrm{R}_{0}\left[\mathrm{abl}-\alpha(1-p) \delta \beta \frac{\Lambda}{\mu \mathrm{R}_{0}}-\mathrm{p} \delta \beta \frac{\Lambda}{\mu \mathrm{R}_{0}} \mathrm{a}-(1-p) \theta \delta \beta \frac{\Lambda}{\mu \mathrm{R}_{0}} \mathrm{~b}\right]+\left(\mathrm{R}_{0}-1\right) a b(\delta+\mu) \mu,
\end{aligned}
$$

and

$$
l=-\left[\beta v \frac{\Lambda}{\mu R_{0}}-(\delta+\mu)\right]>0, \text { since } R_{0}>1 .
$$

Obviously, $b_{1}>0$. By technically complicated calculation, we can obtain

$$
\begin{aligned}
b_{2}= & \mu R_{0}(a+b)+\mu R_{0}(\delta+\mu)+(a+b)(\delta+\mu)+a b-(a+b) \frac{\beta \nu \Lambda}{\mu R_{0}}-p \delta \frac{\beta \Lambda}{\mu R_{0}}-(1-p) \delta \frac{\beta \theta \Lambda}{\mu R_{0}}-\frac{\beta \nu \Lambda}{\mu R_{0}} \\
= & \mu R_{0}(a+b)+a b+\Lambda\left[\frac{(1-p) \delta \theta \beta}{a}+\frac{p \delta \beta}{b}+\frac{\alpha(1-p) \delta \beta}{a b}\right]+\left(1-\frac{1}{R_{0}}\right) \beta \Lambda \nu \\
& +\frac{\Lambda}{\mu R_{0}}\left[\frac{b(1-p) \delta \theta \beta}{a}+\frac{a p \delta \beta}{b}+\frac{\alpha(1-p) \delta \beta}{a b}\right], \\
b_{3}= & \mu R_{0}(a l+a b+b l)-p \delta \beta \Lambda-(1-p) \theta \delta \beta \Lambda+a b\left[(\delta+\mu)-\beta \nu \frac{\Lambda}{\mu R_{0}}\right]-\alpha(1-p) \delta \beta \frac{\Lambda}{\mu R_{0}} \\
& -p \delta \beta \frac{\Lambda}{\mu R_{0}} a-(1-p) \theta \delta \beta \frac{\Lambda}{\mu R_{0}} b+\frac{\beta \Lambda\left(R_{0}-1\right)}{R_{0}}[(a+b) v+p \delta+(1-p) \theta \delta] \\
= & \Lambda(a+b) v \beta\left(1-\frac{1}{R_{0}}\right)+(1-p) \delta \theta \beta \Lambda\left(1+\frac{b}{a}-\frac{1}{R_{0}}\right)+p \delta \beta \Lambda\left(1+\frac{a}{b}-\frac{1}{R_{0}}\right) \\
& +\Lambda(a+b) \frac{\alpha(1-p) \delta \beta}{a b}+\mu R_{0} a b, \\
b_{4}= & a b(\delta+\mu) \mu\left(R_{0}-1\right) .
\end{aligned}
$$

Since $R_{0}>1, b_{2}>0, b_{3}>0$, and $b_{4}>0$ hold, then it follows that all the coefficients of characteristic equation (3.2) are positive. Agian, the Descartes' Sign Rules indicates that all the eigenvalues of (3.2) are negative. Hence, $E_{1}$ is locally stable.

(ii) Secondly, we demonstrate that $E_{1}$ is globally attractive by constructing suitable Lyapunov function and choosing appropriate coefficients. Denote

$$
x=\frac{S}{S^{*}}, \quad y=\frac{E}{E^{*}}, \quad z=\frac{A}{A^{*}}, \quad u=\frac{I}{I^{*}} .
$$

Then system (2.1) can be expressed as

$$
\left\{\begin{array}{l}
x^{\prime}=x\left[\frac{\Lambda}{S^{*}}\left(\frac{1}{x}-1\right)-\beta I^{*}(u-1)-\beta \theta A^{*}(z-1)-\beta v E^{*}(y-1)\right], \\
y^{\prime}=y\left[\beta \frac{S^{*} I^{*}}{E_{1}}\left(\frac{x u}{y}-1\right)+\frac{\beta \theta S^{*} A^{*}}{E^{*}}\left(\frac{x z}{y}-1\right)+\beta v S^{*}(x-1)\right], \\
z^{\prime}=z\left[(1-p) \delta \frac{E^{*}}{A^{*}}\left(\frac{y}{z}-1\right)\right], \\
u^{\prime}=u\left[p \delta \frac{E^{*}}{I^{*}}\left(\frac{y}{u}-1\right)+\alpha \frac{A^{*}}{I^{*}}\left(\frac{z}{u}-1\right)\right] .
\end{array}\right.
$$


Obviously, the endemic equilibrium $E_{1}$ of system (2.1) is equivalent to the positive equilibrium $\bar{E}_{1}=$ $(1,1,1,1)$ of system (3.3). And the global stability of $\bar{E}_{1}$ and $E_{1}$ are the same, hence, we will discuss the global stability of the equilibrium $\bar{E}_{1}$ of system (3.3) instead. In the following, the dynamics of system (3.3) will be analyzed in a bounded feasible region

$$
\Omega=\left\{\bar{X}=(x, y, z, u) \in R_{+}^{4}: x S^{*}+y E^{*}+z A^{*}+u I^{*} \leqslant \frac{\Lambda}{\mu}\right\} .
$$

Define the Lyapunov function

$$
\mathrm{L}=\mathrm{c}_{1} \mathrm{~S}^{*}(x-1-\ln x)+\mathrm{c}_{2} \mathrm{E}^{*}(y-1-\ln y)+\mathrm{c}_{3} A^{*}(z-1-\ln z)+\mathrm{c}_{4} \mathrm{I}^{*}(u-1-\ln u),
$$

where $c_{1}, c_{2}, c_{3}$, and $c_{4}$ are positive and left unspecified. The derivative of $L$ along solutions of system (3.3) is

$$
\begin{aligned}
\mathrm{L}^{\prime}(\mathrm{t})= & c_{1}\left(2 \Lambda-\beta \theta S^{*} A^{*}-\beta S^{*} I^{*}-\beta v S^{*} E^{*}\right)+c_{2}\left(\beta S^{*} I^{*}+\beta \theta S^{*} A^{*}+\beta \nu S^{*} E^{*}\right)+c_{3}(1-p) \delta E^{*} \\
& +c_{4}\left(p \delta E^{*}+\alpha A^{*}\right)+\left(-c_{1} \Lambda+c_{1} \beta S^{*} I^{*}+c_{1} \beta \theta S^{*} A^{*}+c_{1} \beta v S^{*} E^{*}-c_{2} \beta v S^{*} E^{*}\right) x-c_{1} \Lambda \frac{1}{x} \\
& +\left(-c_{1} \beta S^{*} I^{*}+c_{2} \beta S^{*} I^{*}\right) x u+\left(c_{1} \beta S^{*} I^{*}-c_{4} p \delta E^{*}-c_{4} \alpha A^{*}\right) u \\
& +\left(-c_{1} \beta \theta S^{*} A^{*}+c_{2} \beta \theta S^{*} A^{*}\right) x z \\
& +\left[c_{1} \beta \theta S^{*} A^{*}-c_{3}(1-p) \delta E^{*}+c_{4} \alpha A^{*}\right] z+\left(-c_{1} \beta v S^{*} E^{*}+c_{2} \beta v S^{*} E^{*}\right) x y \\
& +\left[c_{1} \beta v S^{*} E^{*}+c_{3}(1-p) \delta E^{*}+c_{4} p \delta E^{*}-c_{2} \beta S^{*} I^{*}-c_{2} \beta \theta S^{*} A^{*}-c_{2} \beta v S^{*} E^{*}\right] y \\
& -c_{3}(1-p) \delta E^{*} \frac{y}{z}-c_{4} p \delta E^{*} \frac{y}{u}-c_{4} \delta A^{*} \frac{z}{u}-c_{2} \beta S^{*} I^{*} \frac{x u}{y}-c_{2} \beta \theta S^{*} A^{*} \frac{x z}{y} \\
= & : F(x, y, z, u) .
\end{aligned}
$$

To determine $c_{1}, c_{2}, c_{3}$, and $c_{4}$ and prove that $F(x, y, z, u) \leqslant 0$, we use the following method to rearrange the items in $F(x, y, z, u)$.

Table 1: The terms that corresponds to $F(x, y, z, u)$.

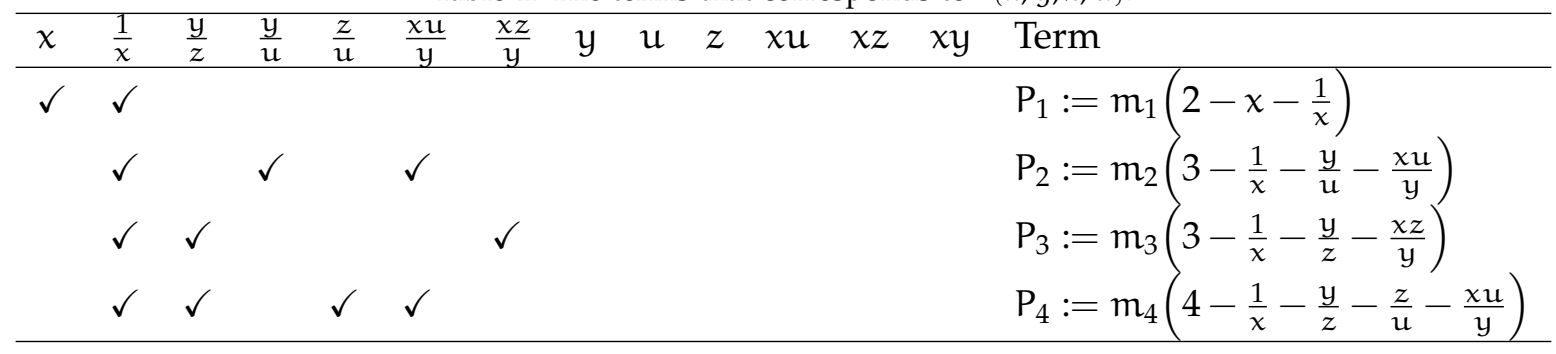

Step 1. Construct a table (Table 1). First, let all the terms appearing in $F(x, y, z, u)$, including $x, \frac{1}{x}, \frac{y}{z}, \frac{y}{u}, \frac{z}{u}$, $\frac{x u}{y}, \frac{x z}{y}, y, u, z, x u, x z, x y$ be the entries in the first row and name them as Term in the last column. Second, from the second row mark with a symbol $\checkmark$ where the product of some of the terms $x, \frac{1}{x}, \frac{y}{z}, \frac{y}{u}, \frac{z}{u}, \frac{x u}{y}$, $\frac{x z}{y}, y, u, z, x u, x z, x y$ equals one, then write the expression for $P_{k}:=m_{k}\left(n_{k}-x_{k, 1}-x_{k, 2}-\cdots-x_{k, n_{k}}\right)$ in the last column, where $\prod_{i=1}^{n_{k}} x_{k, i}=1, n_{k}$ is the number of the terms $x_{k, i}, i=1,2, \cdots, n_{k}, m_{k}$ is a non-negative number and left unspecified. 
Step 2. Let the coefficients of the unmarked terms with symbol $\checkmark$ in Table 1 for function $F(x, y, z, u)$ be zero, that is, the coefficients of $y, u, z, x u, x z$ and $x y$ terms are equal to zero, then we have the following equation

$$
\left\{\begin{array}{l}
c_{1} \beta v S^{*} E^{*}+c_{3}(1-p) \delta E^{*}+c_{4} p \delta E^{*}-c_{2} \beta S^{*} I^{*}-c_{2} \beta \theta S^{*} A^{*}-c_{2} \beta v S^{*} E^{*}=0, \\
c_{1} \beta S^{*} I^{*}-c_{4} p \delta E^{*}-c_{4} \alpha A^{*}=0, \\
c_{1} \beta \theta S^{*} A^{*}-c_{3}(1-p) \delta E^{*}+c_{4} \alpha A^{*}=0, \\
-c_{1} \beta S^{*} I^{*}+c_{2} \beta S^{*} I^{*}=0 \\
-c_{1} \beta \theta S^{*} A^{*}+c_{2} \beta \theta S^{*} A^{*}=0, \\
-c_{1} \beta v S^{*} E^{*}+c_{2} \beta v S^{*} E^{*}=0 .
\end{array}\right.
$$

By simple calculation, we can obtain

$$
c_{2}=c_{1}, \quad c_{3}=\left(\frac{\beta \theta S^{*} A^{*}}{(1-p) \delta E^{*}}+\frac{\beta S^{*} I^{*} \alpha A^{*}}{(1-p) \delta E^{*}\left[p \delta E^{*}+\delta A^{*}\right]}\right) c_{1}, \quad c_{4}=\frac{\beta S^{*} I^{*}}{p \delta E^{*}+\alpha A^{*}} c_{1} .
$$

Set $c_{1}=1$, then Eq. (3.4) admits one set of solutions

$$
c_{1}=1, \quad c_{2}=1, \quad c_{3}=\frac{\beta \theta S^{*} A^{*}}{(1-p) \delta E^{*}}+\frac{\beta S^{*} I^{*} \alpha A^{*}}{(1-p) \delta E^{*}\left[p \delta E^{*}+\delta A^{*}\right]^{\prime}}, \quad c_{4}=\frac{\beta S^{*} I^{*}}{p \delta E^{*}+\alpha A^{*}} .
$$

Given $c_{1}, c_{2}, c_{3}$ and $c_{4}$ above, the Lyapunov function $L$ is positive definite. Substituting the values of $c_{1}$, $c_{2}, c_{3}$ and $c_{4}$ into function $F(x, y, z, u)$ yields

$$
\begin{aligned}
\mathrm{F}(x, y, z, u)= & 2 \Lambda+\beta \theta S^{*} A^{*}+\beta S^{*} I^{*}+\frac{\beta S^{*} I^{*} \alpha A^{*}}{p \delta E^{*}+\alpha A^{*}}-\left(\Lambda-\beta S^{*} I^{*}-\beta \theta S^{*} A^{*}\right) x-\beta \theta S^{*} A^{*} \frac{x z}{y} \\
& -\Lambda \frac{1}{x}-\left(\beta \theta S^{*} A^{*}+\frac{\beta S^{*} I^{*} \alpha A^{*}}{p \delta E^{*}+\alpha A^{*}}\right) \frac{y}{z}-\frac{p \delta E^{*} \beta S^{*} I^{*}}{p \delta E^{*}+\alpha A^{*}} \frac{y}{u}-\frac{\beta S^{*} I^{*} \alpha A^{*}}{p \delta E^{*}+\alpha A^{*}} \frac{z}{u}-\beta S^{*} I^{*} \frac{x u}{y} .
\end{aligned}
$$

Step 3. Let the coefficients for the same terms between $F(x, y, z, u)$ and $\sum_{i=1}^{4} P_{k}$ be equal, then we can obtain the following equations

$$
\begin{aligned}
& \text { the coefficient of } x:-m_{1}=-\left(\Lambda-\beta S^{*} I^{*}-\beta \theta S^{*} A^{*}\right), \\
& \text { the coefficient of } \frac{1}{x}:-m_{1}-m_{2}-m_{3}-m_{4}=-\Lambda, \\
& \text { the coefficient of } \frac{y}{z}:-m_{3}-m_{4}=-\left(\beta \theta S^{*} A^{*}+\frac{\beta S^{*} I^{*} \alpha A^{*}}{p \delta E^{*}+\alpha A^{*}}\right), \\
& \text { the coefficient of } \frac{y}{u}:-m_{2}=-\frac{p \delta E^{*} \beta S^{*} I^{*}}{p \delta E^{*}+\alpha A^{*}}, \\
& \text { the coefficient of } \frac{z}{u}:-m_{4}=-\frac{\beta S^{*} I^{*} \alpha A^{*}}{p \delta E^{*}+\alpha A^{*}}, \\
& \text { the coefficient of } \frac{x u}{y}:-m_{2}-m_{4}=-\beta S^{*} I^{*}, \\
& \text { the coefficient of } \frac{x z}{y}:-m_{3}=-\beta \theta S^{*} A^{*} .
\end{aligned}
$$

Further calculation yields

$$
m_{1}=\beta S^{*} v E^{*}+\mu S^{*}, m_{2}=\frac{p \delta E^{*} \beta S^{*} I^{*}}{p \delta E^{*}+\alpha A^{*}}, m_{3}=\beta \theta S^{*} A^{*}, m_{4}=\frac{\beta S^{*} I^{*} \alpha A^{*}}{p \delta E^{*}+\alpha A^{*}} .
$$

Obviously, $m_{1}, m_{2}, m_{3}$ and $m_{4}$ are all positive values.

Step 4. Compare the constant terms between $F(x, y, z, u)$ and $\sum_{i=1}^{4} P_{k}$ and verify their equality. Applying the equations obtained in Step 3, the equality holds, that is,

$$
2 m_{1}+3 m_{2}+3 m_{3}+4 m_{4}=2 \Lambda+\beta \theta S^{*} A^{*}+\beta S^{*} I^{*}+\frac{\beta S^{*} I^{*} \alpha A^{*}}{p \delta E^{*}+\alpha A^{*}} .
$$


Then by Steps 1-4, function $F(x, y, z, u)$ can be expressed in the following form

$$
\begin{aligned}
F(x, y, z, u)= & \left(\beta S^{*} v E^{*}+\mu S^{*}\right)\left(2-x-\frac{1}{x}\right)+\frac{p \delta E^{*} \beta S^{*} I^{*}}{p \delta E^{*}+\alpha A^{*}}\left(3-\frac{1}{x}-\frac{y}{u}-\frac{x u}{y}\right) \\
& +\beta \theta S^{*} A^{*}\left(3-\frac{1}{x}-\frac{y}{z}-\frac{x z}{y}\right)+\frac{\beta S^{*} I^{*} \alpha A^{*}}{p \delta E^{*}+\alpha A^{*}}\left(4-\frac{1}{x}-\frac{y}{z}-\frac{z}{u}-\frac{x u}{y}\right) .
\end{aligned}
$$

According to that the arithmetic mean is greater than or equal to the geometric mean, there follows $F(x, y, z, u) \leqslant 0$. The equality holds only when $x=1$ and $y=u=z$, that is, $\{(x, y, z, u) \in \Omega: x=1, y=$ $u=z\}$, which is equivalent to the set $\left\{(S, E, A, I) \in \Gamma \mid S=S^{*}, \frac{E}{E^{*}}=\frac{A}{A^{*}}=\frac{I}{I^{*}}\right\} \subset \Gamma$, whose maximum invariant set is the singleton $E_{1}$, then the endemic equilibrium $E_{1}$ is globally asymptotically stable by the LaSalle's invariance principle [18].

As is shown in Fig. 2, if the basic reproduction number $R_{0}=2.210>1$, then the endemic equilibrium $E_{1}$ is globally asymptotically stable.
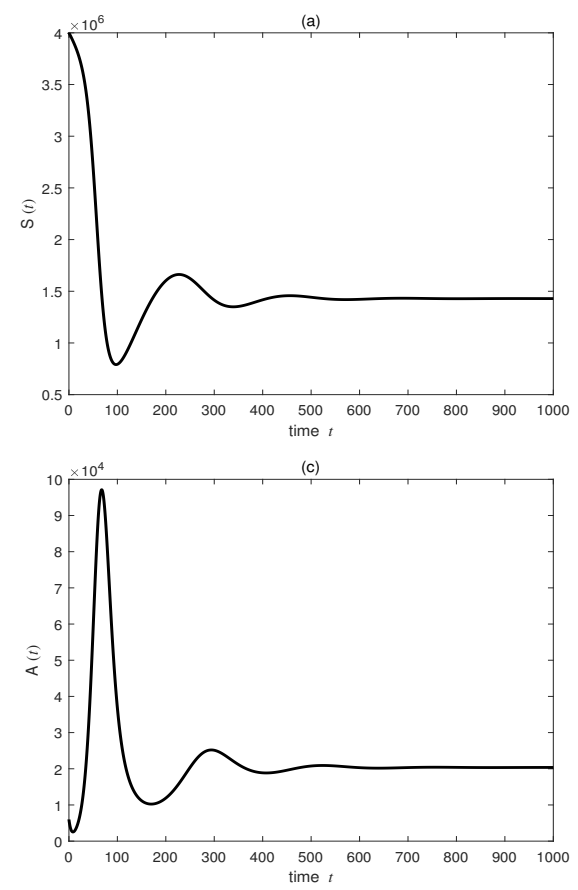

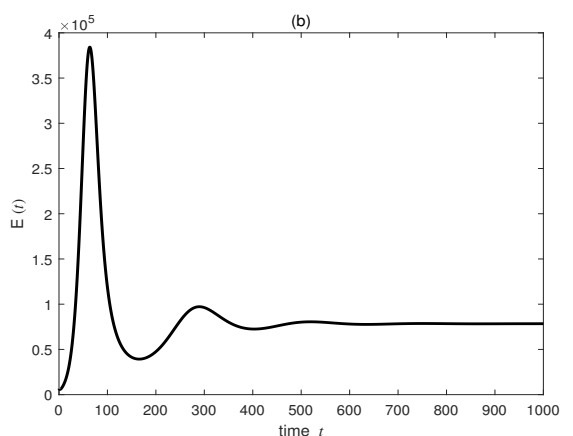

(d)



Figure 2: The endemic equilibrium $E_{1}$ is globally asymptotically stable. $R_{0}=2.210>1 . \Lambda=30000 ; \delta=0.2 ; v=0.032 ; \theta=0.045$; $\mu=0.0095 ; p=0.69 ; \gamma_{1}=0.1292 ; \alpha=0.1 ; \gamma_{2}=0.0978 ; d=0.008$ and $\beta=0.0000001$.

In view of the current situation that the government encourages people to vaccine against COVID-19, we consider immunizing susceptible people based on the system (2.1) in the following.

\section{Model with Pulse vaccination}

Based on system (2.1), we establish the following dynamic model of COVID-19 with pulse vaccination

$$
\left\{\begin{array}{c}
E^{\prime}(t)=\beta S(I+\theta A+v E)-(\delta+\mu) E \\
A^{\prime}(t)=(1-p) \delta E-\left(\gamma_{1}+\alpha+\mu\right) A, \\
I^{\prime}(t)=p \delta E+\alpha A-\left(\gamma_{2}+d+\mu\right) I, \\
S^{\prime}(t)=\Lambda-\beta S(I+\theta A+v E)-\mu S, \\
E\left(n T^{+}\right)=E(n T), A\left(n T^{+}\right)=A(n T), I\left(n T^{+}\right)=I(n T) \\
S\left(n T^{+}\right)=\left(1-p_{1}\right) S(n T)
\end{array}\right.
$$


$R^{\prime}(t)=\gamma_{1} A+\gamma_{2} I-\mu R, R\left(n T^{+}\right)=R(n T)+p_{1} S(n T)$. The proportion $p_{1}$ of the susceptible is vaccinated with time interval $T, n=1,2, \ldots$, and removes to recovered class $R$ permanently. For $R$ can be decoupled from the first four equations, hence we omit it in the following analysis.

We first show that system (4.1) has a disease-free periodic solution. Let $E=A=I=0$, system (4.1) is then simplified to

$$
\left\{\begin{array}{l}
S^{\prime}(t)=\Lambda-\mu S, t \neq n T \\
S\left(n T^{+}\right)=\left(1-p_{1}\right) S(n T), t=n T
\end{array}\right.
$$

According to the variation of constant formula, the solution of system $(4.2)$ on the interval $(n T,(n+1) T]$ is

$$
S(t)=\frac{\Lambda}{\mu}+S\left(n T^{+}\right) e^{-\mu(t-n T)}-\frac{\Lambda}{\mu} e^{-\mu(t-n T)} .
$$

According to the second equation of system (4.2), we get the stroboscopic map

$$
S\left((n+1) T^{+}\right)=\left(1-p_{1}\right) S((n+1) T)=\left(1-p_{1}\right)\left[\frac{\Lambda}{\mu}+S\left(n T^{+}\right) e^{-\mu T}-\frac{\Lambda}{\mu} e^{-\mu T}\right] \triangleq f_{s}\left(S\left(n T^{+}\right)\right) .
$$

The unique fixed point $S S^{*}$ of the map $f_{s}\left(S\left(n T^{+}\right)\right)$is

$$
\mathrm{SS}^{*}=\frac{\Lambda}{\mu}\left[1+\frac{\mathrm{p}_{1}}{\left(1-\mathrm{p}_{1}\right) \mathrm{e}^{-\mu \mathrm{T}}-1}\right]
$$

So the periodic solution of system (4.2) on the interval $(n T,(n+1) T]$ is

$$
\tilde{S}(t)=\frac{\Lambda}{\mu}+S S^{*} e^{-\mu(t-n T)}-\frac{\Lambda}{\mu} e^{-\mu(t-n T)}=\frac{\Lambda}{\mu}+\frac{\Lambda}{\mu} \frac{p_{1}}{\left(1-p_{1}\right) e^{-\mu T}-1} e^{-\mu(t-n T)} .
$$

Hence, the disease-free periodic solution of the system $(4.1)$ is $\tilde{E}(t)=(0,0,0, \tilde{S}(t))$ on every impulsive interval $(n T,(n+1) T], n=1,2, \ldots$

Next we give the definition of the basic reproduction number by applying the next generation operator $[34,35]$. Define two generation matrices at the disease-free periodic solution, that is,

$$
\overline{\mathrm{F}}=\left(\begin{array}{ccc}
v \beta \tilde{S}(\mathrm{t}) & \theta \beta S(t) & \beta \tilde{S}(t) \\
0 & 0 & 0 \\
0 & 0 & 0
\end{array}\right), \quad \bar{V}=\left(\begin{array}{ccc}
\delta+\mu & 0 & 0 \\
-(1-p) \delta & \gamma_{1}+\alpha+\mu & 0 \\
-p \delta & -\alpha & \gamma_{2}+d+\mu
\end{array}\right)
$$

Suppose that $Y(t, s)$ is the evolution operator of system $\frac{d Y}{d t}=-\bar{V} Y(t), t \geqslant s$, and $Y(s, s)=\bar{H}$, where $\bar{H}$ is a $3 \times 3$ identity matrix. Define the next infection operator $\overline{\mathrm{L}}$,

$$
(\overline{\mathrm{L}} \Phi)(\mathrm{t})=\lim _{\mathrm{a} \rightarrow-\infty} \int_{a}^{t} \mathrm{Y}(\mathrm{t}, \mathrm{s}) \overline{\mathrm{F}}(\mathrm{s}) \Psi(\mathrm{s}) \mathrm{ds}, \quad \forall \mathrm{t} \in(\mathrm{nT},(\mathrm{n}+1) \mathrm{T}], \mathrm{n}=1,2, \ldots, \Phi \in \mathrm{C}_{\omega},
$$

where $C_{\omega}$ is defined as the ordered Banach space of all T-periodic functions from $R$ to $R^{3}$, equipped with the maximum norm $\|$. $\|$, and the positive cone $C_{\omega}^{+} \triangleq\left\{\Psi \in C_{\omega}: \Psi(t) \geqslant 0, t \in R\right\}, \Psi$ is the initial distribution of infectious individuals. Then the basic reproduction number of system (4.1) can be defined as the spectral radius of the next infection operator, that is,

$$
\mathrm{R}_{0}=\rho(\overline{\mathrm{L}}) .
$$

\subsection{Global asymptotic stability of the disease-free periodic solution}

For system (4.1), we have the following result for the stability of the disease-free periodic solution $\tilde{E}(t)$.

Theorem 4.1. For system (4.1), there holds, 
(i) $R_{0}=1$ if and only if $\rho\left(\Phi_{\overline{\mathrm{F}}-\overline{\mathrm{V}}}(\mathrm{T})\right)=1$;

(ii) $R_{0}>1$ if and only if $\rho\left(\Phi_{\overline{\mathrm{F}}-\bar{V}}(\mathrm{~T})\right)>1$;

(iii) $R_{0}<1$ if and only if $\rho\left(\Phi_{\overline{\mathrm{F}}-\bar{V}}(T)\right)<1$.

Here, $\Phi_{\overline{\mathrm{F}}-\overline{\mathrm{V}}}(\mathrm{T})$ is the monodromy matrix of $\mathrm{x}^{\prime}=(\overline{\mathrm{F}}-\overline{\mathrm{V}}) \mathrm{x}$. If $\mathrm{R}_{0}<1$, the disease-free periodic solution $\tilde{\mathrm{E}}(\mathrm{t})=$ $(0,0,0, \tilde{S}(t))$ is locally stable; if $\mathrm{R}_{0}>1$, the disease-free periodic solution is unstable.

Proof. The proof of (i)-(iii) can refer to Ref. [33]. Apply Taylor expansion for system (4.1) at the disease-free periodic solution and omit the higher-order terms, we can obtain the following linear system

$$
X^{\prime}(t)=\left(\begin{array}{cc}
\bar{F}-\bar{V} & 0_{3 \times 1} \\
\square & -\mu
\end{array}\right) X(t), t \neq n T, \quad X\left(n T^{+}\right)=\left(\begin{array}{cc}
\bar{H} & 0_{3 \times 1} \\
0_{1 \times 3} & 1-p
\end{array}\right) X(t), t=n T,
$$

where $\square=(-v \beta \tilde{S}(t),-\theta \beta \tilde{S}(t),-\beta \tilde{S}(t))$. The monodromy matrix of system (4.3) is

$$
\left(\begin{array}{cc}
\overline{\mathrm{H}} & 0_{3 \times 1} \\
0_{1 \times 3} & 1-p
\end{array}\right)\left(\begin{array}{cc}
\Phi_{\overline{\mathrm{F}}-\overline{\mathrm{V}}}(\mathrm{T}) & 0 \\
* * & e^{-\mu \mathrm{T}}
\end{array}\right)
$$

where ${ }^{* *}$ is a non-zero vector, $0_{i \times j}$ is a $i \times j$ zero matrix, $i, j=1,3$. Then the Floquet multiplier of the impulse system (4.3) is the maximum of the spectral radius of $\rho\left(\Phi_{\bar{F}-\bar{V}}(T)\right)$ and $(1-p) e^{-\mu T}<1$. Hence, if $\rho\left(\Phi_{\overline{\mathrm{F}}-\bar{V}}(T)\right)<1$, the disease-free periodic solution is locally stable; otherwise, it is unstable.

Lemma 4.2. Let $\mathrm{k}=\frac{1}{\mathrm{~T}} \ln \rho\left(\Phi_{\mathrm{B}(.)}(\mathrm{T})\right)$. Then there exists a positive, $\mathrm{T}$-periodic function $v(\mathrm{t})$ such that $\mathrm{e}^{\mathrm{kt}} v(\mathrm{t})$ is a solution of $\dot{x}=\mathrm{B}(\mathrm{t}) \mathrm{x}, \mathrm{B}(\mathrm{t})=\mathrm{B}(\mathrm{t}+\mathrm{T}), \mathrm{x}: \mathrm{R} \rightarrow \mathrm{R}^{\mathrm{n}}, \mathrm{B}(\mathrm{t})$ is an $\mathrm{n} \times \mathrm{n}$ matrix.

Theorem 4.3. If $\mathrm{R}_{0}<1$, the disease-free periodic solution $\tilde{\mathrm{E}}=(0,0,0, \tilde{\mathrm{S}}(\mathrm{t}))$ of system (4.1) is globally asymptotically stable.

Proof. The local stability of the disease-free periodic solution follows from Theorem 4.1. Next we show that $\tilde{E}$ is globally attractive.

By the uniform and ultimate boundedness of system (2.1) and the non-negativity of the equations, there exists a time $t_{1}$ and a sufficiently small positive number $\epsilon_{1}$, such that when $t>t_{1}, S(t) \leqslant \frac{\Lambda}{\mu}+\epsilon_{1}$ holds. From the first three equations of system (4.1), when $t>t_{1}$, we have

$$
\left\{\begin{array}{l}
E^{\prime}(t) \leqslant \beta\left(\frac{\Lambda}{\mu}+\epsilon_{1}\right)(I+\theta A+\nu E)-(\delta+\mu) E, \\
A^{\prime}(t)=(1-p) \delta E-\left(\gamma_{1}+\alpha+\mu\right) A, \\
I^{\prime}(t)=p \delta E+\alpha A-\left(\gamma_{2}+d+\mu\right) I .
\end{array}\right.
$$

Denote $z(t)=(E(t), A(t), I(t))^{\top}$, consider the following comparison system

$$
z^{\prime}(\mathrm{t})=\left(\overline{\mathrm{F}}-\overline{\mathrm{V}}+\mathrm{M}\left(\epsilon_{1}\right)\right) z(\mathrm{t}),
$$

where

$$
M\left(\epsilon_{1}\right)=\left(\begin{array}{ccc}
v \beta \epsilon_{1} & \theta \beta \epsilon_{1} & \beta \epsilon_{1} \\
0 & 0 & 0 \\
0 & 0 & 0
\end{array}\right) .
$$

By Lemma 4.2, there exists a positive function $v(t)=\left(v_{1}(t), v_{2}(t), v_{3}(t)\right)^{\top}$ with $T$ as the period such that $z(t)=e^{k_{1} t} v(t)$ is a solution of system (4.5), where $\kappa_{1}=\frac{1}{T} \ln \rho\left(\Phi_{\bar{F}}-\bar{V}+M\left(\epsilon_{1}\right)(T)\right)$. Since $\epsilon_{1}$ is small enough and $\rho\left(\Phi_{\bar{F}-\bar{V}+M\left(\epsilon_{1}\right)}(T)\right)$ is continuous with respect to $\epsilon_{1}$, so that $\rho\left(\Phi_{\bar{F}-\bar{V}+M\left(\epsilon_{1}\right)}(T)<1\right.$, that is $\kappa_{1}<0$. So when $t \rightarrow \infty, z(t) \rightarrow 0$, for initial value $\left(E\left(t_{1}\right), A\left(t_{1}\right), I\left(t_{1}\right)\right)^{\top}$ of system (4.4), there exists a sufficiently large $z^{*}$ such that $\left(E\left(t_{1}\right), A\left(t_{1}\right), I\left(t_{1}\right)\right)^{\top} \leqslant z^{*}\left(v_{1}(0), v_{2}(0), v_{3}(0)\right)^{\top}$. The comparison theorem then indicates that

$$
(E(t), A(t), I(t))^{\top} \leqslant z^{*} e^{k_{1}\left(t-t_{1}\right)}\left(v_{1}\left(t-t_{1}\right), v_{2}\left(t-t_{1}\right), v_{3}\left(t-t_{1}\right)\right)^{\top}, t \geqslant t_{1},
$$

when $t \rightarrow \infty, E(t) \rightarrow 0, A(t) \rightarrow 0, I(t) \rightarrow 0$. The Theorem is proved. stable.

As is shown in Fig. 3, if $R_{0}<1$, then the disease-free periodic solution $\tilde{E}$ is globally asymptotically 



Figure 3: The disease-free periodic solution $\tilde{E}$ is globally asymptotically stable. $R_{0}=0.837 . T=14 ; p_{1}=0.01 ; \Lambda=30000 ; \delta=0.2 ;$ $v=0.032 ; \theta=0.045 ; \mu=0.0095 ; p=0.69 ; \gamma_{1}=0.1292 ; \alpha=0.1 ; \gamma_{2}=0.0978 ; d=0.008 ;$ and $\beta=0.00000002$.

\subsection{Persistence of system (4.1)}

Denote

$$
X=R_{+}^{4}, \quad X_{0}=\{(E, A, I, S) \in X: S>0\}, \quad \partial X \triangleq X \backslash X_{0} .
$$

Define Poincaré map $\mathrm{P}: \mathrm{R}_{+}^{4} \rightarrow \mathrm{R}_{+}^{4}, \forall x^{0} \in X, \mathrm{P}\left(x^{0}\right)=\mathrm{u}\left(\mathrm{T}, \mathrm{x}^{0}\right), \mathrm{u}\left(\mathrm{t}, \mathrm{x}^{0}\right)$ is the unique solution of system (4.1) and satisfies $u\left(0, x^{0}\right)=x^{0}$. It is easy to verify that $\left.P^{m}\left(E^{0}, A^{0}, I^{0}, S^{0}\right)=u\left(m T, E^{0}, A^{0}, I^{0}, S^{0}\right)\right), \forall m \geqslant 0$. Poincaré map $P$ has a unique fixed point $E^{*}=(0,0,0, \tilde{S}(0))$ in $X$. We first give the following lemma.

Lemma 4.4. If $\mathrm{R}_{0}>1$, there exists a positive constant $\delta_{0}>0$, such that for all $x^{0}=\left(\mathrm{E}^{0}, \mathrm{~A}^{0}, \mathrm{I}^{0}, \mathrm{~S}^{0}\right) \in \mathrm{X}_{0}$, $\left\|x^{0}-E^{*}\right\| \leqslant \delta_{0}$, we have

$$
\limsup _{m \rightarrow \infty} d\left(P^{m}\left(x^{0}\right), E E^{*}\right) \geqslant \delta_{0} .
$$

Proof. By the continuity of the solutions with respect to the initial values, for any $\varepsilon_{1}>0$, there exists a $\delta_{0}>0$ such that for all $x^{0} \in X_{0}$ with $\left\|x^{0}-E E^{*}\right\| \leqslant \delta_{0}$, there holds

$$
\left\|u\left(t, x^{0}\right)-u\left(t, E E^{*}\right)\right\| \leqslant \varepsilon_{1}, \forall t \in[0, T] .
$$

Suppose Eq. (4.6) is not satisfied, then for some $x^{0} \in X_{0}$,

$$
\limsup _{m \rightarrow \infty} d\left(P^{m}\left(x^{0}\right), E E^{*}\right)<\delta_{0}
$$

Without loss of generality, suppose $d\left(P^{m}\left(x^{0}\right), E E^{*}\right)<\delta_{0}, \forall m>0$. For any $t \geqslant 0$, let $t=\underline{m} T+t^{\prime}$, where $t^{\prime} \in[0, T]$ and $\underline{m}=\left[\frac{t}{T}\right]$ is the greatest integer less than or equal to $\frac{t}{T}$. Hence, we get

$$
\left\|u\left(t, x^{0}\right)-u\left(t, E E^{*}\right)\right\|=\left\|u\left(t^{\prime}, P \underline{m}\left(x^{0}\right)\right)-u\left(t^{\prime}, E E^{*}\right)\right\|<\varepsilon_{1}, \forall t \geqslant 0 .
$$


It follows that $0 \leqslant E(t) \leqslant \varepsilon_{1}, 0 \leqslant A(t) \leqslant \varepsilon_{1}$, and $0 \leqslant I(t) \leqslant \varepsilon_{1}$. Then by the fourth equation of system (4.1), we get

$$
\left\{\begin{array}{l}
S^{\prime}(t) \geqslant \Lambda-\beta S(1+\theta+v) \varepsilon_{1}-\mu S \\
S\left(n T^{+}\right)=\left(1-p_{1}\right) S(n T)
\end{array}\right.
$$

Consider an auxiliary system

$$
\left\{\begin{array}{l}
\mathrm{S}^{\prime}(\mathrm{t})=\Lambda-\beta S(1+\theta+v) \varepsilon_{1}-\mu \mathrm{S} \\
\mathrm{S}\left(\mathrm{nT}^{+}\right)=\left(1-\mathrm{p}_{1}\right) \mathrm{S}(\mathrm{nT})
\end{array}\right.
$$

System (4.7) has a globally asymptotically stable solution

$$
\hat{S}\left(t, \varepsilon_{1}\right)=\frac{\Lambda}{\beta \varepsilon_{1}(1+\theta+v)+\mu}+\frac{\Lambda}{\beta \varepsilon_{1}(1+\theta+v)+\mu} \frac{p_{1}}{\left(1-p_{1}\right) e^{-\left(\beta \varepsilon_{1}(1+\theta+v)+\mu\right) T}-1} e^{-\left(\beta \varepsilon_{1}(1+\theta+v)+\mu\right)(t-n T)} .
$$

For $\hat{S}\left(t, \varepsilon_{1}\right) \rightarrow \tilde{S}(t)$ as $\varepsilon_{1} \rightarrow 0$, hence, for any $\eta>0$, there exists a $\varepsilon_{2}>0$ and a time $t_{2}$, such that when $\varepsilon_{1}<\varepsilon_{2}$ and $t>t_{2}$, we have $\hat{S}\left(t, \varepsilon_{1}\right) \geqslant \tilde{S}(t)-\eta$. By the comparison theorem, there exists a time $t_{3}>t_{2}$ such that $S(t) \geqslant \hat{S}\left(t, \varepsilon_{1}\right) \geqslant \tilde{S}(t)-\eta, t \geqslant t_{3}$. Now, for the first three equations of system (4.1), when $t \geqslant t_{3}$, there holds

$$
\left\{\begin{array}{l}
E^{\prime}(t) \geqslant \beta(\tilde{S}(t)-\eta)(I+\theta A+v E)-(\delta+\mu) E \\
A^{\prime}(t)=(1-p) \delta E-\left(\gamma_{1}+\alpha+\mu\right) A \\
I^{\prime}(t)=p \delta E+\alpha A-\left(\gamma_{2}+d+\mu\right) I
\end{array}\right.
$$

Consider the comparison system of system (4.8)

$$
\left\{\begin{array}{l}
\hat{E}^{\prime}(t)=\beta(\tilde{S}(t)-\eta)(I+\theta A+v E)-(\delta+\mu) E \\
\hat{A}^{\prime}(t)=(1-p) \delta E-\left(\gamma_{1}+\alpha+\mu\right) A \\
\hat{I}^{\prime}(t)=p \delta E+\alpha A-\left(\gamma_{2}+d+\mu\right) I
\end{array}\right.
$$

By Lemma 4.2, there exists a positive T-periodic function $p(t)=\left(p_{1}(t), p_{2}(t), p_{3}(t)\right)$ such that $e^{k_{2} t} p(t)$ is a solution of system (4.9), where $\kappa_{2}=\frac{1}{T} \ln \rho\left(\Phi_{\bar{F}(\eta)-\bar{V}}(T)\right)$. Since $R_{0}>1$, choose a sufficiently small $\eta$ such that $\rho\left(\Phi_{\bar{F}(\eta)-\bar{V}}(T)\right)>1$, that is $k_{2}>0$. Let $t=\bar{n} T>t_{3}$, where $\bar{n}$ is a positive integer, thus

$$
(\hat{\mathrm{E}}(\overline{\mathrm{n}} T), \hat{\mathrm{A}}(\overline{\mathrm{n}} \mathrm{T}), \hat{\mathrm{I}}(\overline{\mathrm{n}} \mathrm{T}))^{\top}=e^{\mathrm{K}_{2}\left(\overline{\mathrm{n}} \mathrm{T}-\mathrm{t}_{3}\right)}\left(\mathrm{p}_{1}\left(\overline{\mathrm{n}} \mathrm{T}-\mathrm{t}_{3}\right), \mathrm{p}_{2}\left(\overline{\mathrm{n}} \mathrm{T}-\mathrm{t}_{3}\right), \mathrm{p}_{3}\left(\overline{\mathrm{n}} \mathrm{T}-\mathrm{t}_{3}\right)\right)^{\top} \rightarrow(\infty, \infty, \infty)^{\top}, \overline{\mathrm{n}} \rightarrow \infty .
$$

For any initial value $\left(E\left(t_{3}\right), A\left(t_{3}\right), I\left(t_{3}\right)\right)^{\top}$ of system (4.8), there exists a sufficiently small positive $z_{*}$ such that $\left(E\left(t_{3}\right), A\left(t_{3}\right), I\left(t_{3}\right)\right)^{\top} \geqslant z_{*}\left(p_{1}(0), p_{2}(0), p_{3}(0)\right)^{\top}$. According to the comparison theorem, when $t \geqslant t_{3}$,

$$
(E(t), A(t), I(t))^{\top} \geqslant z_{*} e^{k_{2}\left(t-t_{3}\right)}\left(\hat{E}\left(t-t_{3}\right), \hat{A}\left(t-t_{3}\right), \hat{I}\left(t-t_{3}\right)\right)^{\top} .
$$

Hence

$$
\lim _{t \rightarrow \infty} E(t)=\infty, \lim _{t \rightarrow \infty} A(t)=\infty, \lim _{t \rightarrow \infty} I(t)=\infty,
$$

which contradicts with the boundedness of the solutions, thus the lemma is established.

Theorem 4.5. If $\mathrm{R}_{0}>1$, there is a positive constant $\varepsilon>0$, for all initial values $\left(\mathrm{E}^{0}, \mathrm{~A}^{0}, \mathrm{I}^{0}, \mathrm{~S}^{0}\right) \in \mathrm{R}_{+}^{4}$, the solutions of system (4.1) satisfy $\lim _{t \rightarrow \infty}(\mathrm{E}(\mathrm{t}), \mathrm{A}(\mathrm{t}), \mathrm{I}(\mathrm{t}), \mathrm{S}(\mathrm{t}))>(\varepsilon, \varepsilon, \varepsilon, \varepsilon)$, that is, system (4.1) is uniformly persistent, and there exists at least one positive periodic solution.

For the convenience of description, we present the following persistence theory, [36, Theorem 1.3.1].

Lemma 4.6. Assume that

(C1) $\mathrm{P}\left(\mathrm{X}_{0}\right) \subset \mathrm{X}_{0}$ and $\mathrm{P}$ has a global attractor $\mathrm{AT}$; 
(C2) the maximal compact invariant set $\mathrm{A}_{\partial}=\mathrm{AT} \cap \mathrm{M}_{\partial}$ of $\mathrm{P}$ in $\partial \mathrm{X}_{0}$, possibly empty, admits a Morse decomposition $\left\{M_{1}, \ldots, M_{k}\right\}$ with the following properties:

(a) $M_{i}$ is isolated in $X$;

(b) $W^{s}\left(M_{i}\right) \cap X_{0}=\varnothing$ for each $1 \leqslant i \leqslant k$.

Then there exists $\delta>0$ such that for any compact internally chain transitive set LL with $L L \nsubseteq M_{i}$ for all $1 \leqslant i \leqslant k$, we have $\inf _{\mathrm{x} \in \mathrm{LL}} \mathrm{d}\left(\mathrm{x}, \partial \mathrm{X}_{0}\right)>\delta$, that is to say, $\mathrm{P}: \mathrm{X} \rightarrow \mathrm{X}$ is uniformly persistent with respect to $\left(\mathrm{X}_{0}, \partial \mathrm{X}_{0}\right)$.

Proof of Theorem 4.5. Now we prove that $P$ is uniformly persistent with respect to $\left(X_{0}, \partial X_{0}\right)$. It can be seen from system (4.1) that both $X$ and $X_{0}$ are positively invariant and $\partial X_{0}$ is a relatively closed set in $X$. By the ultimate and uniform boundedness of the solutions of system (4.1), the Poincaré map P admits a global attractor [34, 35]. Let

$$
M_{\partial}=\left\{\left(E^{0}, A^{0}, I^{0}, S^{0}\right) \in \partial X_{0}: P^{m}\left(\left(E^{0}, A^{0}, I^{0}, S^{0}\right)\right) \in \partial X_{0}, \forall m \geqslant 0\right\}
$$

And next we prove that

$$
M_{\partial}=\{(0,0,0, S): S \geqslant 0\} .
$$

Suppose $\left(E^{0}, A^{0}, I^{0}, S^{0}\right) \in \partial X_{0} \backslash\{(0,0,0, S): S \geqslant 0\}$. Without loss of generality, take $I^{0}=0, E^{0} A^{0} \neq 0$. By the third equation of system (4.1), we have

$$
I^{\prime}(t)=p \delta E^{0}+\alpha A^{0}>0
$$

For the continuity of the solution, when $t>0$ is sufficiently small, $(E, A, I, S) \notin \partial X_{0}$, that is, $(E, A, I, S) \notin$ $M_{\partial}$. The inverse proposition can show that formula (4.10) holds.
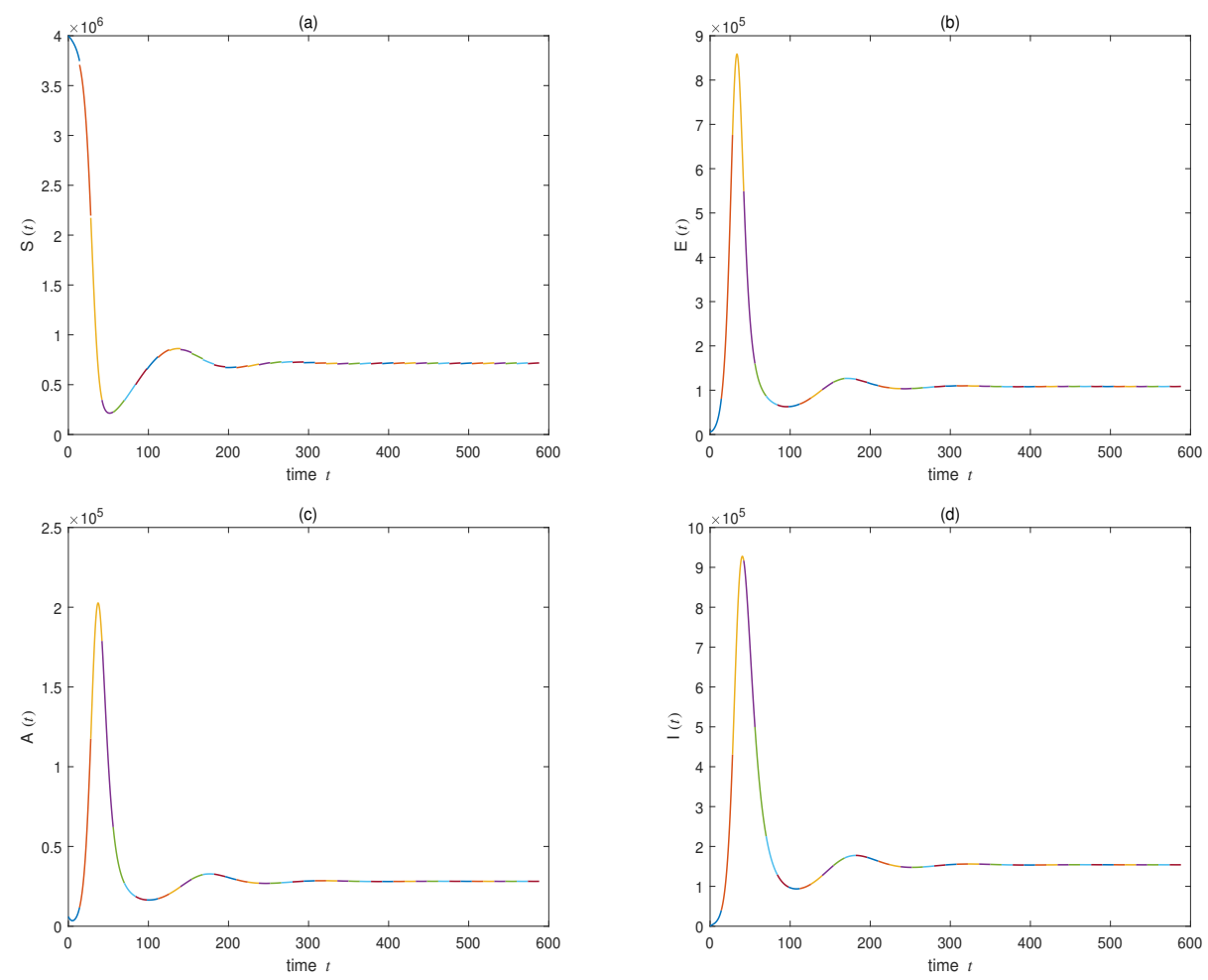

Figure 4: The disease is uniformly persistent if $R_{0}>1 . R_{0}=2.38 . T=14 ; p_{1}=0.01 ; \Lambda=30000 ; \delta=0.2 ; \nu=0.032 ; \theta=0.045$; $\mu=0.0095 ; p=0.69 ; \gamma_{1}=0.1292 ; \alpha=0.1 ; \gamma_{2}=0.0978 ; d=0.008$; and $\beta=0.0000002$.

On the other hand, $\mathrm{P}$ has only one fixed point $\mathrm{EE}^{*}=(0,0,0, \tilde{\mathrm{S}}(0))$ in $\mathrm{M}_{\partial}$. By Lemma 4.4, the invariant set $E^{*}$ is isolated, and $W^{s}\left(E E^{*}\right) \cap X_{0}=\varnothing$. The orbits in $M_{\partial}$ all converge to $E E^{*}$, and $E^{*}$ is acyclic in 
$M_{\partial}$. According to the conclusion in $[1-4,23-25,36], P$ is uniformly persistent with respect to $\left(X_{0}, \partial X_{0}\right)$, so that the solution of system (4.1) is uniformly persistent with respect to $\left(X_{0}, \partial X_{0}\right)$. Theorem 1.3.6 in [36] further states that $P$ has a fixed point $x^{*} \in X_{0}$, so that the solution of system (4.1) passing through $x^{*}$ is a non-trivial periodic solution and $E(t)>0, A(t)>0, I(t)>0$. The theorem is proved.

As is shown in Fig. 4, if $R_{0}>1$, the system (4.1) is uniformly persistent.

\section{Conclusion}

Based on the two key features of COVID-19 pandemic, that is, the infectiveness of the incubation period and the infected showing no symptoms, we proposed a SEAIR epidemic model in this paper. By applying an algebraic approach, we could express the derivative of the Lyapunov function as sums of $m_{k}\left(n_{k}-x_{k, 1}-x_{k, 2}-\cdots-x_{k, n_{k}}\right)$ with $\prod_{i=1}^{n_{k}} x_{k, i}=1$, hence, due to the property that the arithmetic mean is greater than or equal to the geometric mean, we could obtain the derivative is negative. What follows is the disease free equilibrium is globally asymptotically stable if $R_{0}<1$, while the endemic equilibrium is globally asymptotically stable if $R_{0}>1$. As far as we know, few models related to COVID-19 give the global dynamics of the disease. The Lyapunov function method presented in our model could be extended to more general models with COVID-19.

We further considered pulse vaccination for COVID-19 and established an impulsive system. We defined the basic reproduction number as the spectral radius of the next infection operator, and proved that if the basic reproduction number is smaller than 1, the disease-free periodic solution is globally asymptotically stable. Meanwhile, we obtained the uniform persistence of the disease if the basic reproduction number is larger than one by applying the persistent theory.

\section{References}

[1] P. Agarwal, R. P. Agarwal, M. Ruzhansky, Special Functions and Analysis of Differential Equations, Chapman and Hall/CRC, New York, (2020). 4.2

[2] P. Agarwal, D. Baleanu, Y. Q. Chen, S. Momani, J. A. T. Machado, Fractional Calculus, Springer, Singapore, (2018).

[3] P. Agarwal, S. Deniz, S. Jain, A. A. Alderremy, S. Aly, A new analysis of a partial differential equation arising in biology and population genetics via semi analytical techniques Physica A: Statistical Mechanics and its Applications, Physisa A, 542 (2020), 12 pages.

[4] P. Agarwal, S. S. Dragomir, M. Jleli, B. Samet, Advances in Mathematical Inequalities and Applications, Birkhäuser/Springer, Singapore, (2018). 4.2

[5] P. Agarwal, J. J. Nieto, M. Ruzhansky, D. F. M. Torres, Analysis of Infectious Disease Problems (Covid-19) and Their Global Impact, Springer Singapore, Singapore, (2021). 1

[6] A. Atangana, Modelling the spread of COVID-19 with new fractal-fractional operators: Can the lockdown save mankind before vaccination?, Chaos Solitons Fractals, 136 (2020), 38 pages.

[7] A. Atangana, S. I. Araz, Mathematical model of COVID-19 spread in Turkey and South Africa: theory, methods, and applications, Adv. Difference Equ., 2020 (2020), 89 pages.

[8] A. Atangana, S. I. Araz, Modeling and forecasting the spread of COVID-19 with stochastic and deterministic approaches: Africa and Europe, Adv. Difference Equ., 2021 (2021), 107 pages.

[9] I. A. Baba, A. Yusuf, K. S. Nisar, A. H. Abdel-Aty, T. A. Nofal, Mathematical model to assess the imposition of lockdown during COVID-19 pandemic, Results Phys., 20 (2021), 10 pages. 1

[10] L. Basnarkov, SEAIR Epidemic spreading model of COVID-19, Chaos Solitons Fractals, 142 (2021), 15 pages. 1

[11] L. D. Domenico, G. Pullano, C. E. Sabbatini, P. Y. Boelle, V. Colizza, Expected impact of reopening schools after lockdown on COVID-19 epidemic in Ile-de-France, Nat. Commun.,12 (2021), 12 pages. 1

[12] G. Giordano, F. Blanchini, R. Bruno, P. Colaneri, A. Di Filippo, A. Di Matteo, M. Colaneri, Modelling the COVID-19 epidemic and implementation of population-wide interventions in Italy, Nat. Med., 26 (2020), 1-6. 1

[13] X. He, E. H. Y. Lau, P. Wu, X. L. Deng, J. Wang, X. X. Hao, Y. C. Lau, J. Y. Wong, Y. J. Guan, X. G. Tan, X. N. Mo, Y. Q. Chen, B. L. Liao, W. L. Chen, F. G. Hu, Q. Zhang, M. Q. Zhong, Y. R. Wu, L. Z. Zhao, F. C. Zhang, B. J. Cowling, F. Li, G. M. Leung, Temporal dynamics in viral shedding and transmissibility of COVID-19, Nat. Med., 26 (2020), 672-675. 1

[14] G. Hussain, T. Khan, A. Khan, M. Inc, G. Zaman, K. S. Nisar, A. Akgül, Modeling the dynamics of novel coronavirus (COVID-19) via stochastic epidemic model, Alexandria Eng. J., 60 (2021), 4121-4130. 1

[15] H. K. Khalil, Nonlinear Systems, Prentice-Hall, Upper Saddle River, (2002). 1 
[16] A. Korobeinikov, Lyapunov functions and global stability for SIR and SIRS epidemiological models with non-linear transmission, Bull. Math. Biology, 30 (2006), 615-626. 1

[17] A. Korobeinikov, P. K. Maini, Non-linear incidence and stability of infectious disease models, Math. Med. Biol., 22 (2005), 113-128. 1

[18] J. P. LaSalle, The Stability of Dynamical Systems, in: Regional Conference Series in Applied Mathematics, SIAM, Philadelaphia, (1976). 1, 3.1, 3.2

[19] S. A. Lauer, K. H. Grantz, Q. Bi, F. K. Jones, Q. Zheng, H. R. Meredith, A. S. Azman, N. G. Reich, J. Lessler, The incubation period of coronavirus disease 2019 (COVID-19) from publicly reported confirmed cases: estimation and application, Ann. Intern. Med., 172 (2020), 577-582. 1

[20] X. Li, M. Bohner, C. Wang, Impulsive differential equations: Periodic solutions and applications, Automatica, 52 (2015), 173-178.

[21] J. Q. Li, X. Xie, Y. M. Chen, A new way of constructing Lyapunov functions with application to an SI epidemic model, Appl. Math. Lett., 113 (2021), 5 pages. 1

[22] J. Q. Li, Y. L. Yang, Y. C. Zhou, Global stability of an epidemic model with latent stage and vaccination, Nonlinear Anal. Real World Appl., 12 (2011), 2163-2173. 1

[23] A. Rehman, R. Singh, P. Agarwal, Modeling, analysis and prediction of new variants of covid-19 and dengue co-infection on complex network, Chaos Solitons Fractals, 150 (2021), 19 pages. 1, 4.2

[24] M. Ruzhansky, Y. J. Cho, P. Agarwal, I. Area, Advances in Real and Complex Analysis with Applications, Birkhäuser/Springer, Singapore, (2017).

[25] S. Salahshour, A. Ahmadian, N. Senu, D. Baleanu, P. Agarwal, On Analytical Solutions of the Fractional Differential Equation with Uncertainty: Application to the Basset Problem, Entropy, 17 (2015), 885-902 4.2

[26] A. S. Shaikh, I. N. Shaikh, K. Nisar, A mathematical model of COVID-19 using fractional derivative: outbreak in India with dynamics of transmission and control, Adv. Difference Equ., 2020 (2020), 19 pages. 1

[27] B. Shulgin, L. Stone, Z. Agur, Pulase Vaccination Strategy in the SIR Epidemic Model, Bull. Math. Biol., 60 (1998), 1123-1148. 1

[28] L. Stone, B. Shulgin, Z. Agur, Theoretical examination of the pulse vaccination policy in the SIR epidemic model, Math. Compu. Modelling, 31 (2000), 207-215. 1

[29] B. Tang, X. Wang, Q. Li, N. L. Bragazzi, S. Tang, Y. Xiao, J. Wu, Estimation of the Transmission Risk of the 2019nCoV and Its Implication for Public Health Interventions, J. Clin. Med., 9 (2020), 13 pages. 1

[30] R. ud Din, A. R. Seadawy, K. Shah, A. Ullah, D. Baleanu, Study of global dynamics of COVID-19 via a new mathematical model, Results Phys., 19 (2020), 13 pages. 1

[31] P. van den Driessche, J. Watmough, Reproduction numbers and sub-threshold endemic equilibria for compartmental models of disease transmission, Math. Biosc., 180 (2002), 29-48. 2

[32] Y. Wang, M. M. Lu, J. Liu, Global stability of a delayed virus model with latent infection and Beddington-DeAngelis infection function, Appl. Math. Lett., 107 (2020), 9 pages. 1

[33] W. D. Wang, X.-Q. Zhao, Threshold Dynamics for Compartmental Epidemic Models in Periodic Environments, J. Dynam. Differential Equations, 20 (2008), 699-717. 1, 4.1

[34] Y. Yang, Y. Xiao, Threshold dynamics for an HIV model in periodic environments, J. Math. Anal. Appl., 361 (2010), 59-68. 4, 4.2

[35] Y. P. Yang, Y. Xiao, The effects of population dispersal and pulse vaccination on disease control, Math. Comput. Modelling, 52 (2010), 1591-1604. 4, 4.2

[36] X.-Q. Zhao, Dynamical Systems in Population Biology, Springer-Verlag, New York, (2003). 1, 4.2, 4.2 